\title{
Molecular docking analyses of thiazolidine-2,4-dione analogues for PPAR-gamma agonism in the search of antidiabetic agents
}

\section{Santosh Chhajed, Vandana Jadhav and Harshada Mahajan and S.J. Kshirsagar \\ Department of Pharmaceutical Chemistry MET's Institute of Pharmacy, BKC, Nashik, Maharashtra, India-422003}

Abstract: Thiazolidine-2,4-dione acting as an agonist to this receptor PPARgamma, they decrease insulin resistance in adipose tissue, skeletal muscles and liver. In order design useful agent it is quite necessary to understand molecular dynamics of the ligands with their targets. An important aspect of glitazones is acidic head group connected to lipophilic tail by a phenoxyalkyl For rational design of newer ligands, in present work mapping of target PPARy is reported, such that interactions of pharmacophore present on the structure and further exploration of the molecules is possible and helpful for the followers.

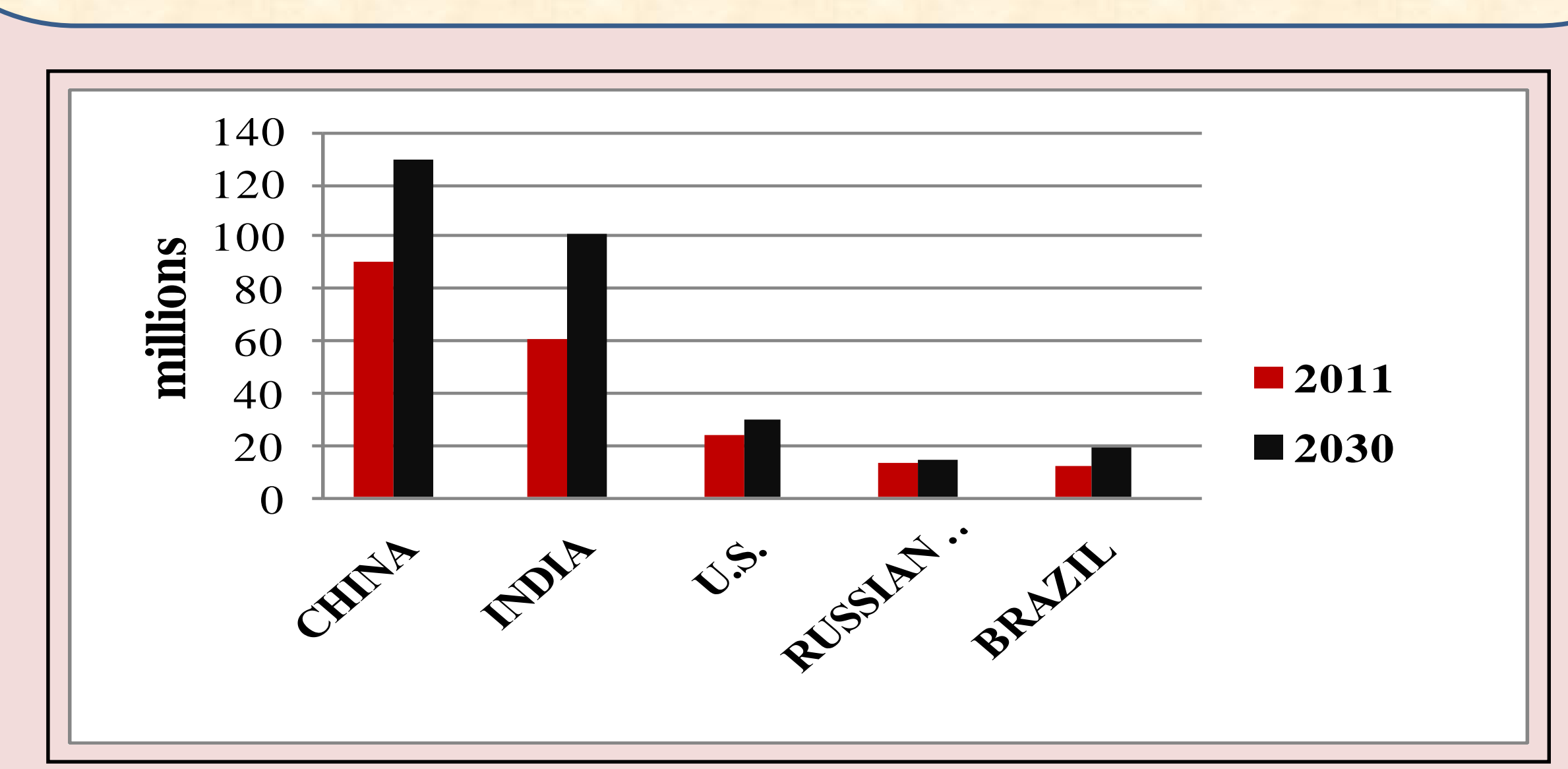

Figure 1| Top countries for estimated population with diabetes

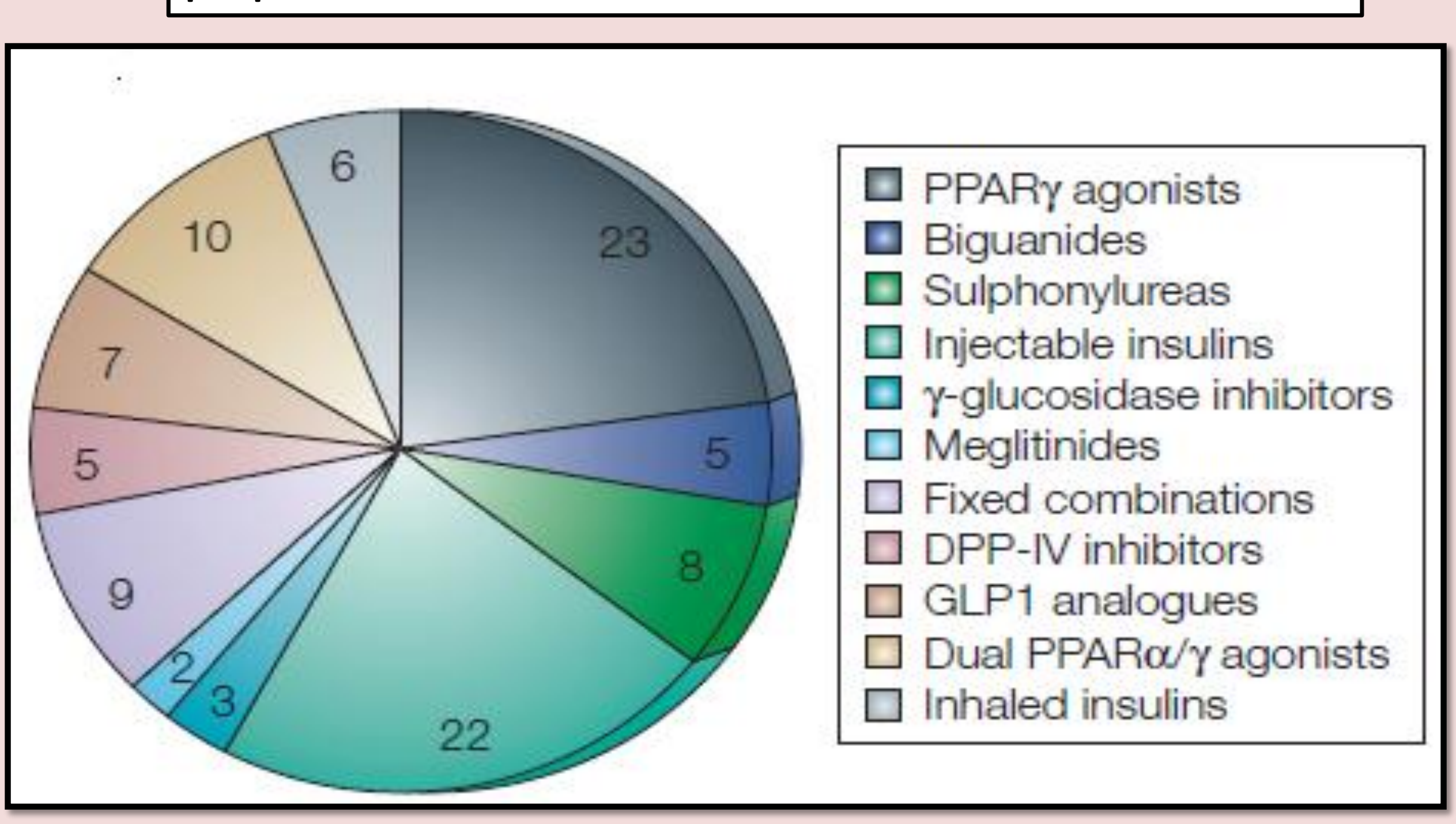

Figure 2| Drug classes contribute to type 2 diabetes market growth (frequently prescribed) DPP: dipeptidyl peptidase GLP1: glucagon-like peptide

\section{Earlier work: Literature}

$>$ Acidic head and hydrophobic tail are essential for activity

$>$ Phenoxyalkyl linker is required as spacer

$>$ Bioisosteric replacement of " $O$ " by " $S$ " results active agents

$>$ Acyl-amide linker $\left(-\mathrm{CH}_{2} \mathrm{CONH}_{2}\right)$ yields active agents

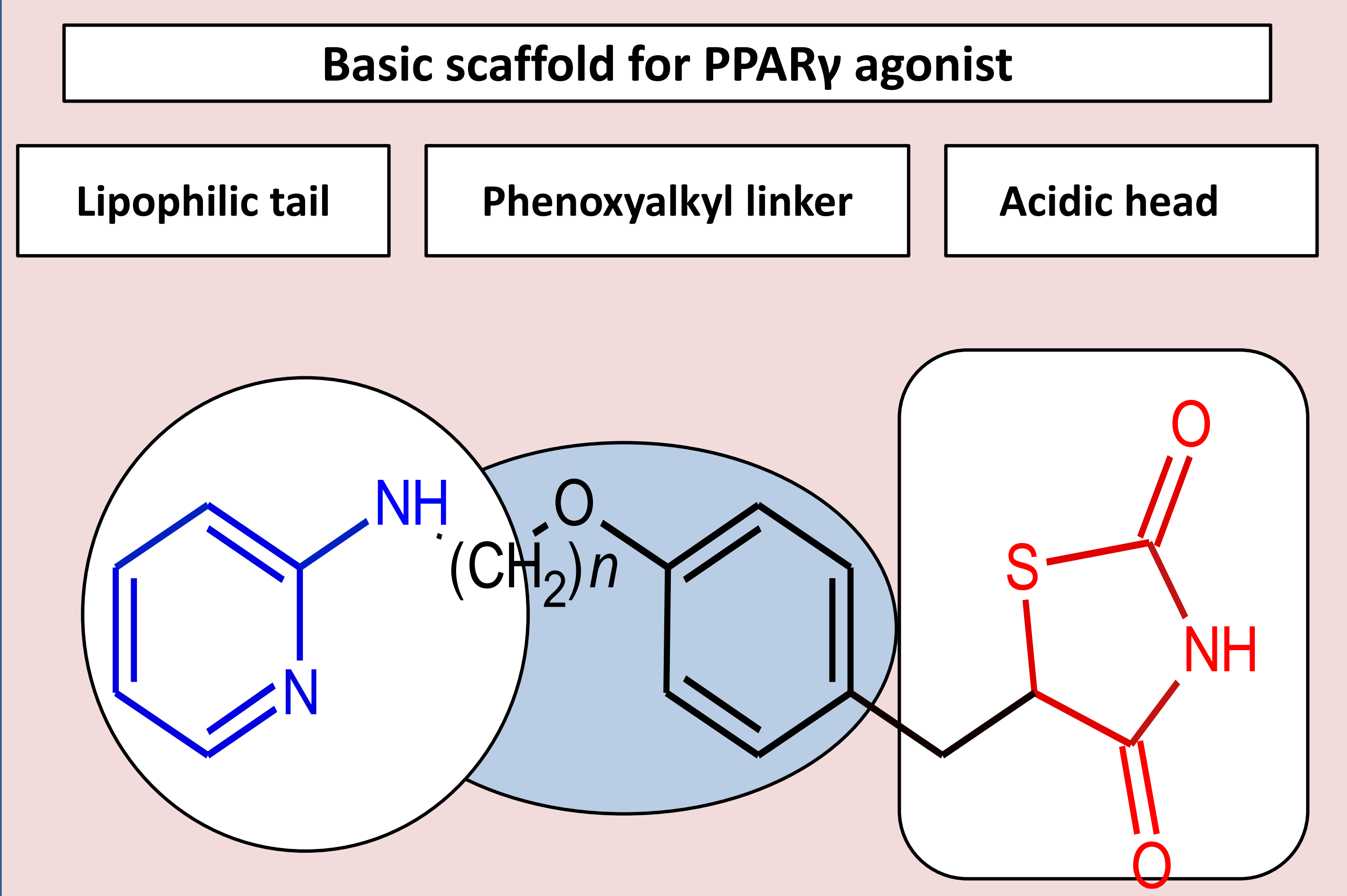

Active Site Mapping

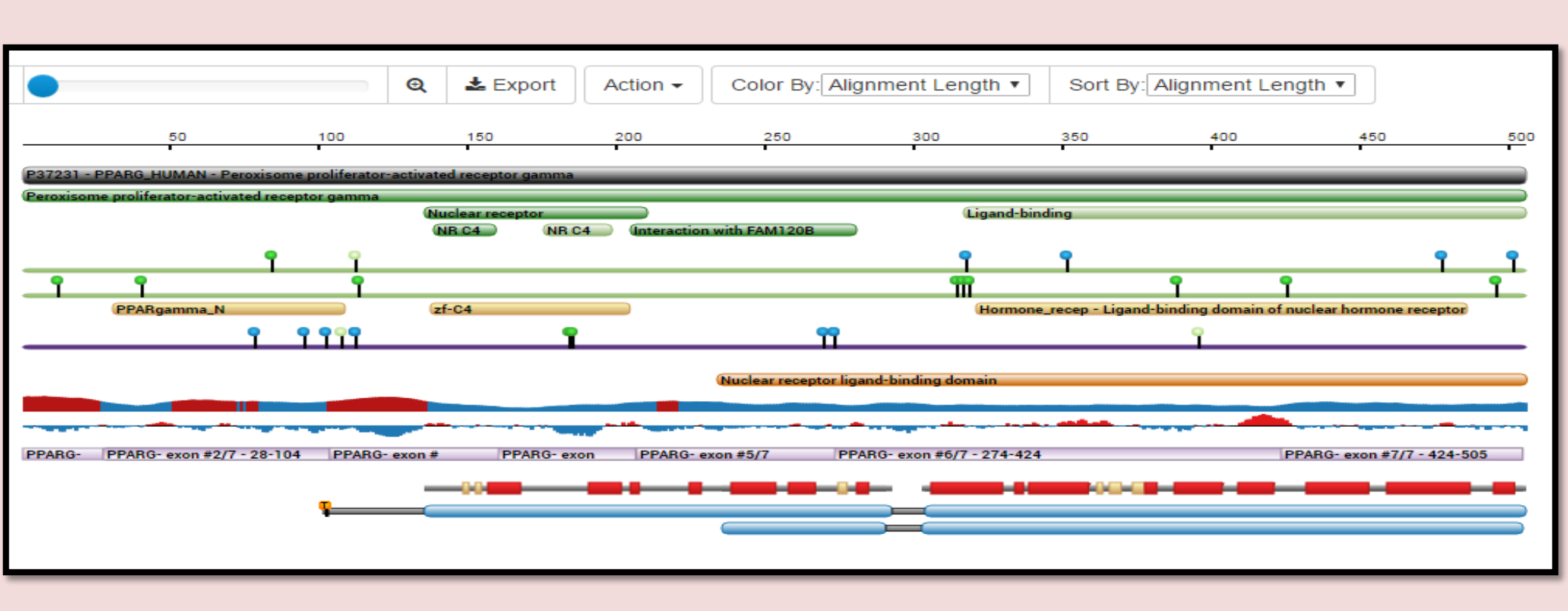

Figure 2| Analysis of protein PPARY by UNIPROT SERVER

"Y" Shaped cavity of PPAR Y

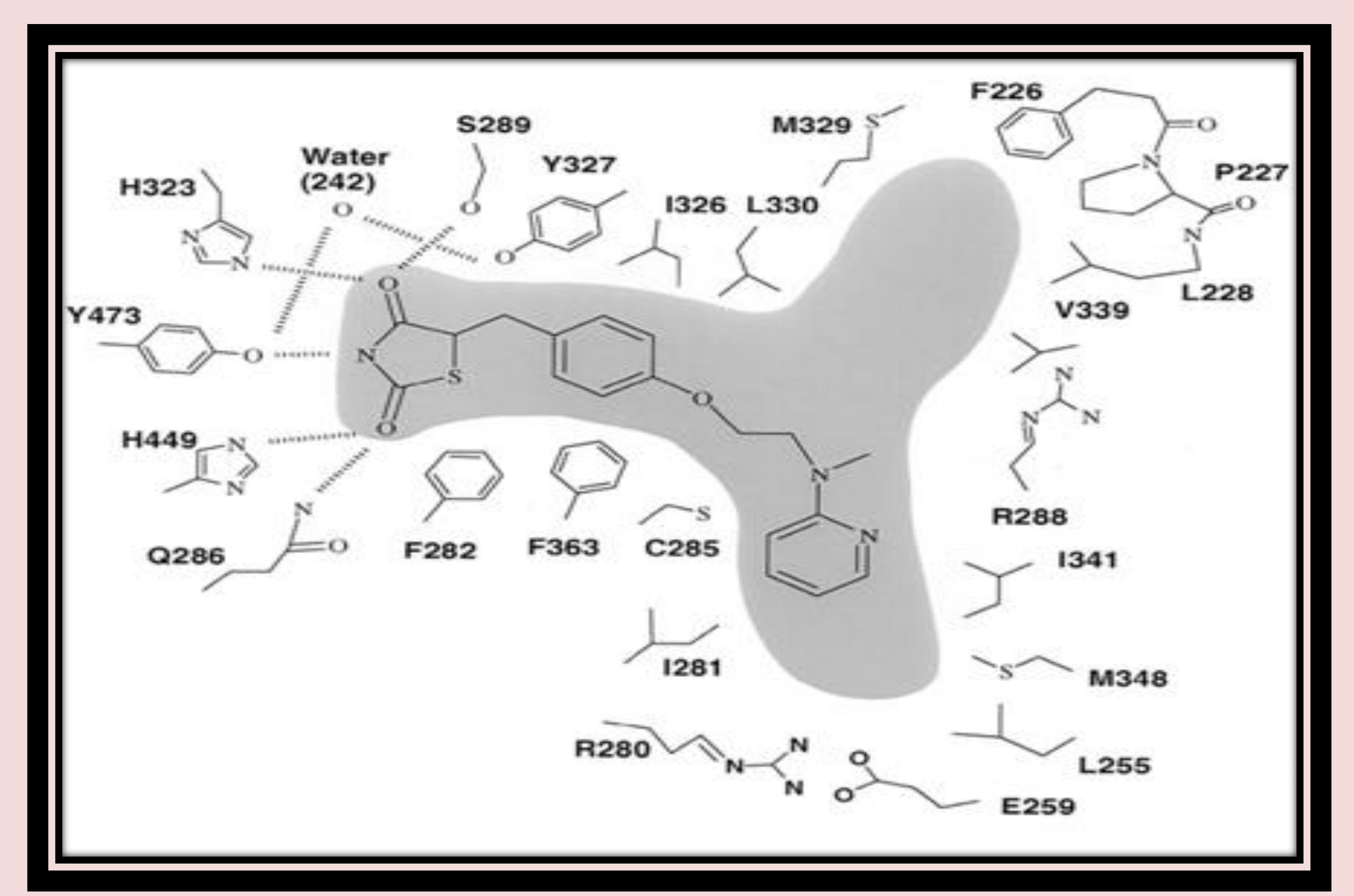

Figure 3| LBD of PPARY is " $\mathrm{Y}$ " shaped cavity, the key hydrogen Figure 3| LBD of PPARY is " $Y$ " shaped cavity, the key hydrogen
bonds are formed between acidic head pieces of rosiglitazone a ful agonist and His and Ser 22914286, Ser289, Ala292, He326, Leu330, Met364, ILe281, ILe324, Gly259, Leu255

FURTHER INSIGHTS: HYDROPHOBICITY SCALE

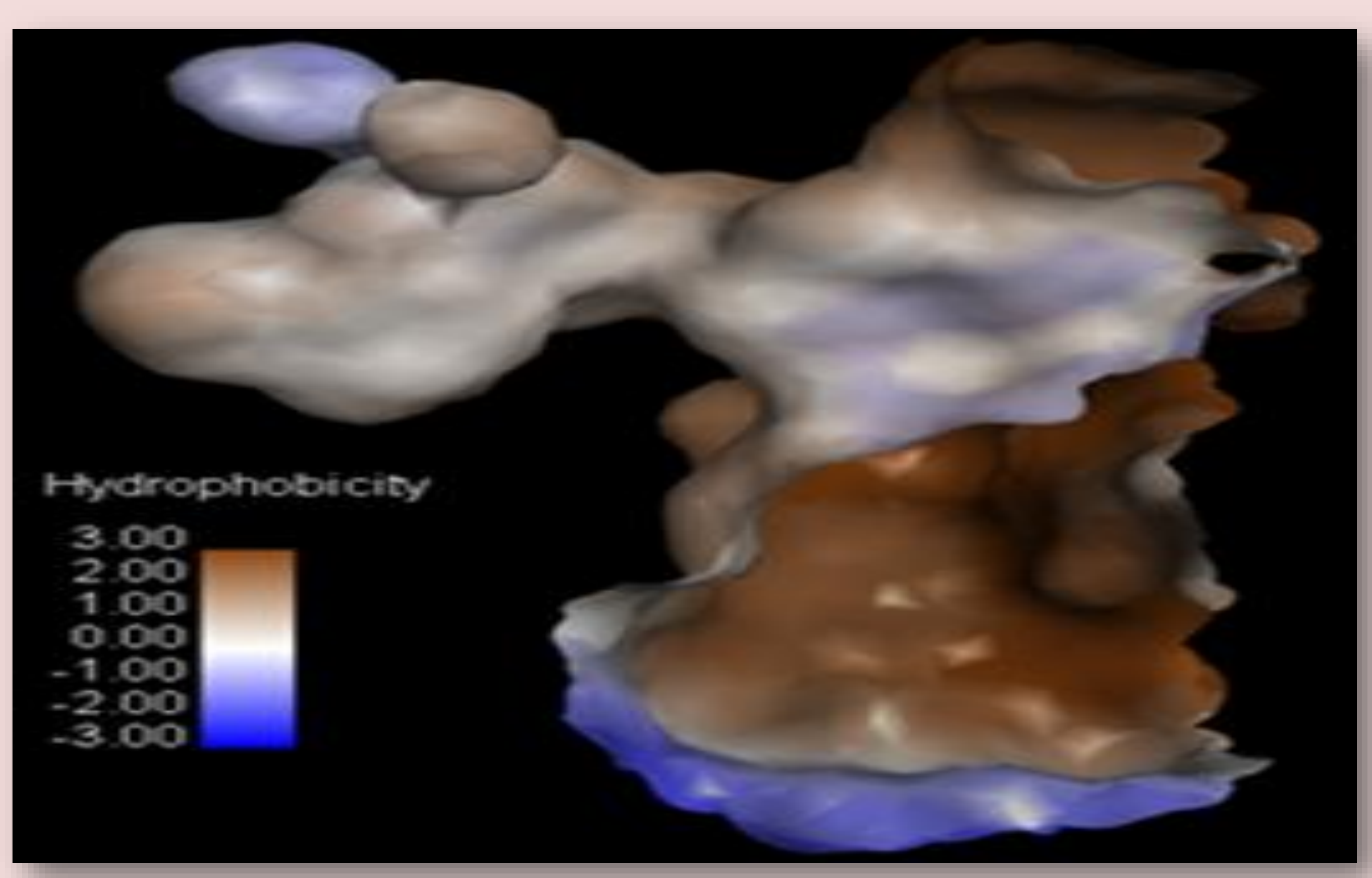

Figure 4| active site of PPARY studied Part of cavity showing BROWN COLOR indicates hydrophobic nature

\section{Highlights of the work}

1) Study of molecular biology of protein PPARgamma

2) Study of active site of PPAR (mapping)

3) To study binding modes (and hence amino acid residues) for existing drug

4) To design novel analogues

5) To perform docking analysis of analogues

\section{De-Novo Design}

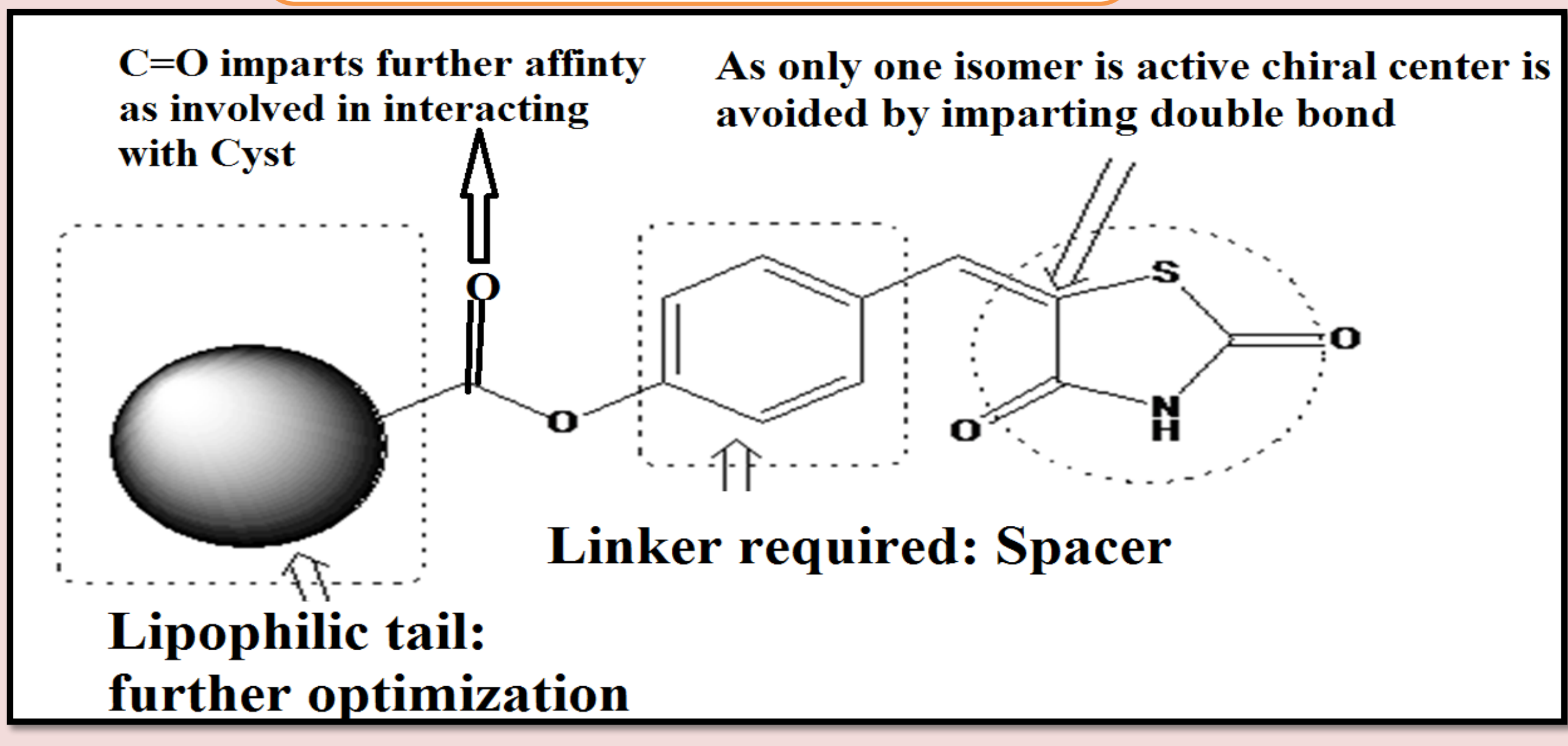

further optimization

Figure 5| Design strategies for novel ligand based on need for binding in to LBD

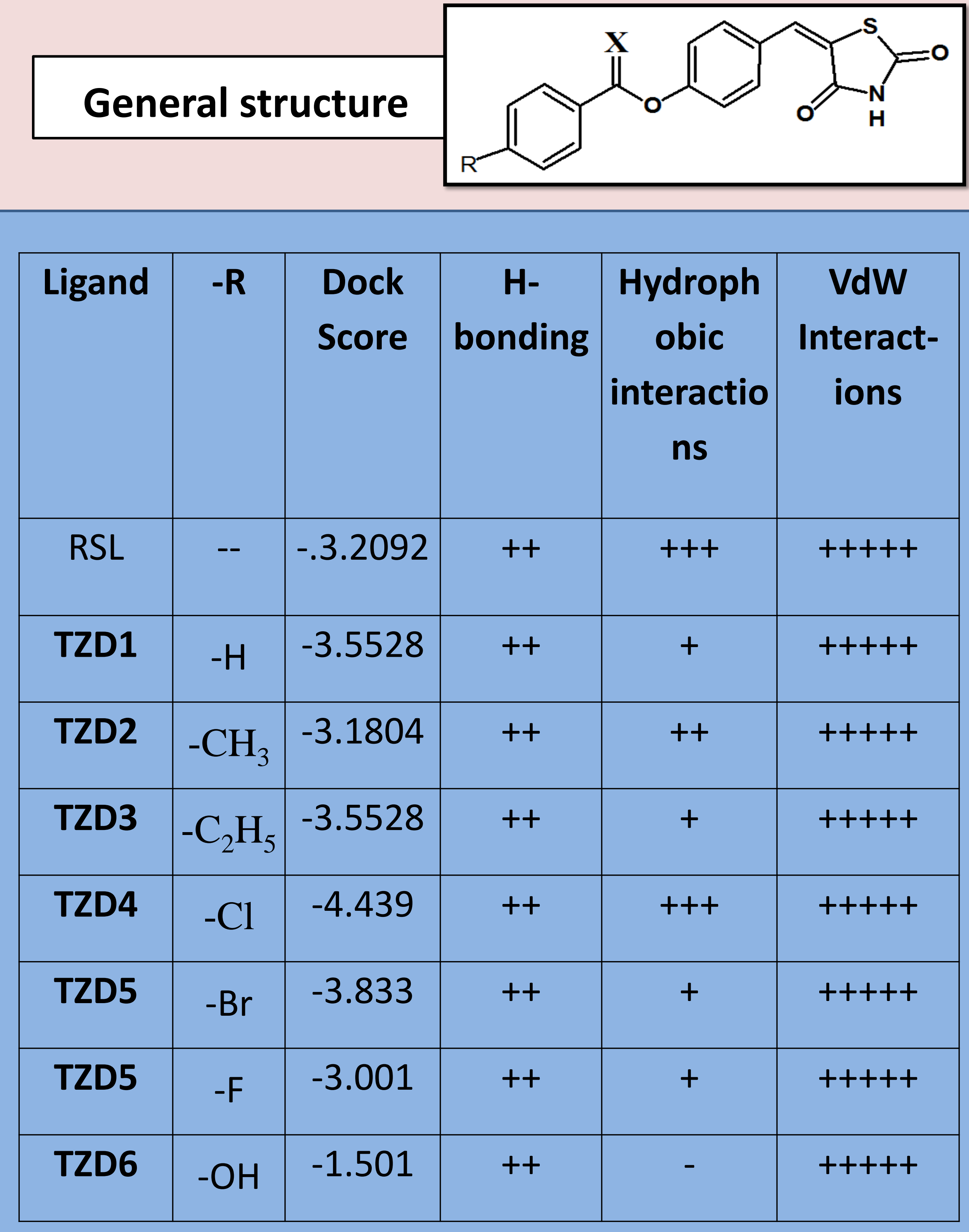

Table 1| Docking analyses of representative analogues

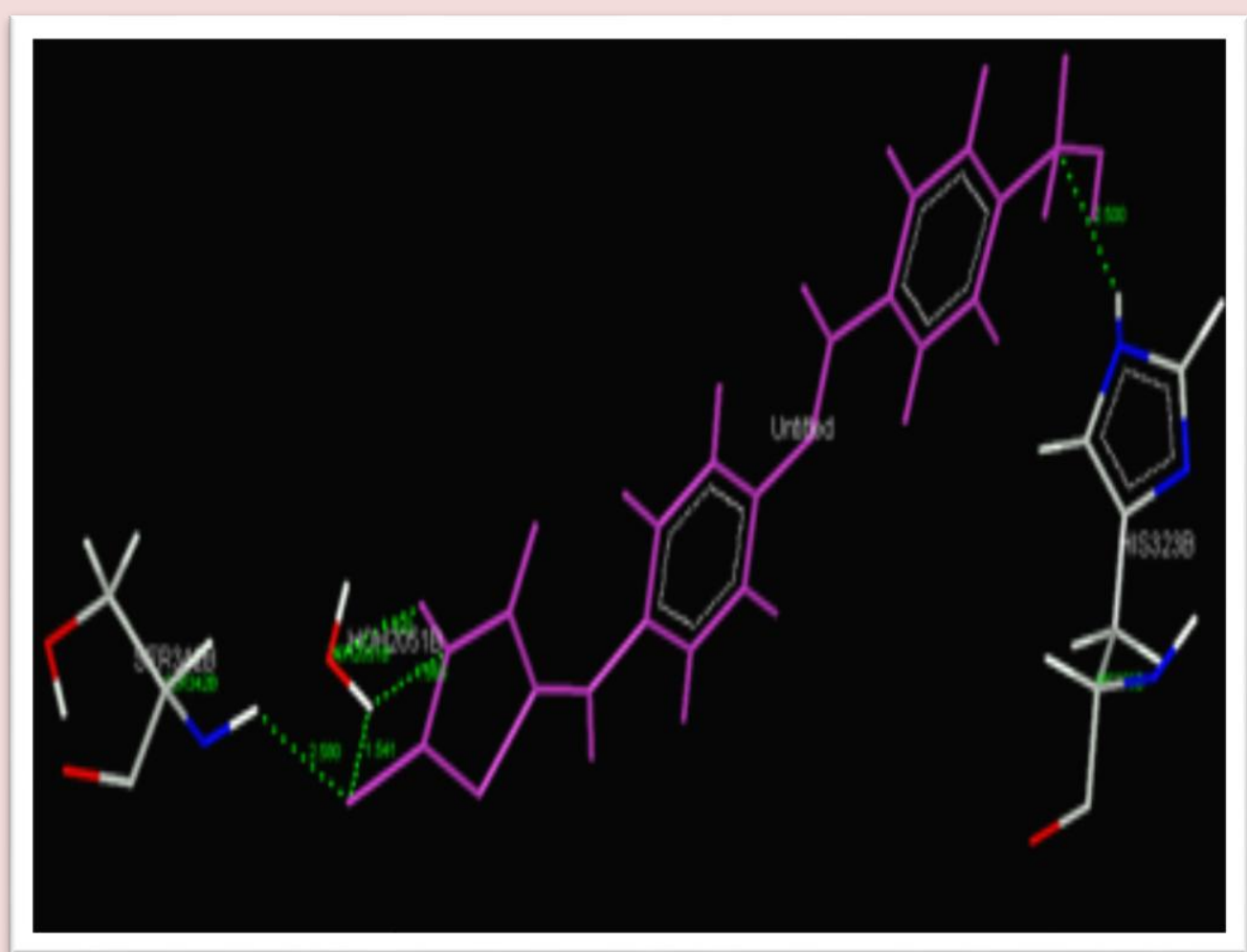

Fig.6(a): Molecule TZD2 in to the active site of PPARY showin

Fig.6(b): TZD1 in to the active site showing 285: importance of $\mathrm{C}=0$ -

Further Studies: Synthesis of designed compounds followed by subject to suitable anti- diabetic assay is necessary towards validation of above studies

Representative References:

1) Abraham, J. D., (Ed.), "Burger's Medicinal Chemistry and Drug Discovery-", VI (ed.), 2003, 27- 30

2) Ottow, E., (Ed.), "Nuclear Receptors as Drug Targets", Wiley- VCH, Germany, 2008. p. no. 367- 81

3) Kota, B. P.; Huang, T. H. and Roufogalis, B. D, (Ed.), "An overview on biological mechanisms of PPARs", Pharmacol. Res. 51(2005) 85- 94 